\title{
Resource Allocation for Relay Assisted Cognitive Radio Networks $\diamond$
}

\author{
Ammar Zafar*, Yunfei Chen^, Mohamed-Slim Alouini*, and Redha M. Radaydeh* \\ *Division of Physical Sciences and Engineering, King Abdullah University of Science and Technology (KAUST) \\ Thuwal, Mekkah Province, Saudi Arabia \\ Emails:\{ammar.zafar, slim.alouini, redha.radaydeh\}@kaust.edu.sa \\ ${ }^{\star}$ School of Engineering, University of Warwick \\ Coventry, UK, CV4 7AL \\ Email: yunfei.chen@warwick.ac.uk
}

\begin{abstract}
In this paper, we present two optimal resource allocation schemes that maximize throughput and symbol correct rate (SCR). The throughput and SCR are derived. The derived throughput and SCR are optimized with respect to the sensing time, the source transmission power and the relay transmission power. Numerical results show that the optimal sensing time is dependent on the primary user's signal-to-noise-ratio (SNR). They also show that SCR increases with increase in the number of relays.
\end{abstract}

\section{INTRODUCTION}

Recent years have seen the employment of cognitive relays to improve cognitive radio performance [1], [2]. In cognitive relaying, secondary nodes act as relays for the source. In this scenario, the source first looks for "spectral holes" using spectrum sensing. Upon detection of a "spectral hole", it transmits information to the destination and the relays. The relays then forward the received signal to the destination to achieve diversity gain.

The secondary user has to balance two conflicting objectives of reducing interference to the primary user and increasing its own throughput. Hence, the secondary user must efficiently employ spectrum sensing so as not to interfere with primary user and also not to miss any transmission slot. The trade off between probability of detection and probability of false alarm can be thought of as the trade off between throughput of the secondary user and the interference to the primary.

In [3], the authors investigated this sensing-throughput trade off for a cognitive radio network without relays and optimized the sensing time for maximization of the throughput. Power allocation strategies for relay assisted cognitive radio networks have been studied in [4]-[6]. However all of these assume co-existence of the primary and secondary networks without spectrum sensing. In other words, the proposed schemes work in an "underlay" setup by keeping a measure of performance, generally the interference experienced by the primary user, below a certain threshold.

In this paper, we consider a relay assisted cognitive radio network with opportunistic access to licensed bands. Sensing is performed only at the transmitting secondary user and not

\footnotetext{
${ }^{\diamond}$ This work was funded by King Abdullah University of Science and Technology (KAUST).
}

at the relays. Two power allocation schemes that maximize throughput and symbol correct rate (SCR) of the secondary source employing cognitive relays are considered. The source divides its power between spectrum sensing and transmission whereas the relays only use their power for forwarding. In addition to individual power constraints, we also consider a global power constraint on the whole system. We assume complete channel state information (CSI). Finally, we will consider amplify-and-forward (AF) relays.

Numerical results show that the optimal power allocation is independent of the number of relays but is dependent on the channel. They also show that the throughput decreases with the number of relays, while the SCR increases. In addition, results show that the optimal sensing time is significantly influenced by the primary user's signal-to-noise-ratio $(S N R)$.

\section{System MODEL}

Consider a system with a source node and a destination node, and $m$ relays between them, as shown in figure 1 . We assume here an orthogonal system. For our discussion, we assume time orthogonality. In the first time slot, the source senses for any primary activity. On detection of a spectral hole the source transmits data to the destination through the relays. The relays amplify the signal from the source and forward it to the destination. Each relay transmission takes place in a different time slot. Thus one packet requires a total of $m+1$ time slots.

With this setup, there are four possible scenarios: 1) The source, with probability of detection $P_{d}$, correctly detects transmission from the primary and will not transmit, 2) The source, with probability $1-P_{d}$, does not detect the transmission from the primary and transmits and as such cause interference, 3) The source, with probability $P_{f}$, incorrectly detects transmission from the primary and stays silent, therefore missing an opportunity for transmission, 4) The source, with probability $1-P_{f}$, correctly detects a "spectral hole" and transmits data to the destination through the $m$ relays.

According to these four scenarios, there are two transmitted signal models. 


\section{A. Without Interference}

The signal at the destination from the source is

$$
y_{r_{t}}=\sqrt{E_{S T}} h_{r_{t}} s+n_{r_{t}}
$$

where $s$ is the zero-mean and unit-energy transmitted symbol, $E_{S T}$ is the energy used by the source for transmission, $h_{r_{t}}$ is the known channel response between the source and the destination and $n_{r_{t}} \sim C N\left(0, \sigma_{n_{r_{t}}}^{2}\right)$ is the complex Gaussian noise. The signal at the $i$ th relay from the source is

$$
y_{i_{t}}=\sqrt{E_{S T}} h_{i_{t}} s+n_{i_{t}}
$$

where $h_{i_{t}}$ is the known channel response between the source and the $i$ th relay and $n_{i_{t}} \sim C N\left(0, \sigma_{n_{i_{t}}}^{2}\right)$ is the complex Gaussian noise. The relay normalizes the received signal and transmits it to the destination. The signal transmitted by the $i$ th relay is

$$
s_{i}=\sqrt{\frac{E_{S T}}{E_{S T}\left|h_{i_{t}}\right|^{2}+\sigma_{n_{i_{t}}}^{2}}} h_{i_{t}} s+\sqrt{\frac{1}{E_{S T}\left|h_{i_{t}}\right|^{2}+\sigma_{n_{i_{t}}}^{2}}} n_{i_{t}} .
$$

Hence the signal at the destination from the $i$ th relay is

$$
y_{r_{i}}=\sqrt{E_{i}} h_{r_{i}} s_{i}+n_{r_{i}}
$$

where $h_{r_{i}}$ is the known channel response between the destination and the $i$ th relay, $E_{i}$ is the energy used for transmission by the $i$ th relay and $n_{r_{i}} \sim C N\left(0, \sigma_{n_{r_{i}}}^{2}\right)$ is the complex Gaussian noise. Putting in $s_{i}$, we can write

$$
y_{r_{i}}=\sqrt{\frac{E_{S T} E_{i}}{E_{S T}\left|h_{i_{t}}\right|^{2}+\sigma_{n_{i_{t}}}^{2}}} h_{r_{i}} h_{i_{t}} s+\tilde{n_{r_{i}}}
$$

where $\tilde{n_{r_{i}}} \sim C N\left(0, \tilde{n_{r_{i}}}\right)$ and

$$
\sigma_{n_{r_{i}}}^{\tilde{2}}=\frac{E_{i}\left|h_{r_{i}}\right|^{2} \sigma_{n_{i_{t}}}^{2}}{E_{S T}\left|h_{i_{t}}\right|^{2}+\sigma_{n_{i_{t}}}^{2}}+\sigma_{n_{r_{i}}}^{2} .
$$

In matrix form one has

$$
\mathbf{y}=\mathbf{h} s+\mathbf{n}
$$

where

$$
\begin{aligned}
& \mathbf{y}=\left[\frac{1}{\sigma_{n_{r_{t}}}} y_{r_{t}} \frac{1}{\sigma_{n_{r_{1}}}^{\sim}} y_{r_{1}} \ldots \ldots \ldots \frac{1}{\tilde{\sigma_{n_{r_{m}}}}} y_{r_{m}}\right]^{T} \\
& \mathbf{h}=\left[\sqrt{\frac{E_{S T}}{\sigma_{n_{r_{t}}}^{2}}} h_{r_{t}} \sqrt{\frac{E_{S T} E_{1}}{\sigma_{n_{r_{1}}}^{\tilde{2}}\left(E_{S T}\left|h_{1_{t}}\right|^{2}+\sigma_{n_{1_{t}}}^{2}\right)}} h_{r_{1}} h_{1_{t}} \ldots\right. \\
& \left.\sqrt{\frac{E_{S T} E_{m}}{\sigma_{n_{r_{m}}}^{2}\left(E_{S T}\left|h_{m_{t}}\right|^{2}+\sigma_{n_{m_{t}}}^{2}\right)}} h_{r_{m}} h_{m_{t}}\right]^{T}
\end{aligned}
$$

and $\mathbf{n} \sim C N(\mathbf{0}, \mathbf{I})$.

\section{B. With Interference}

Now the signal at the destination from the source is

$$
y_{r_{t}}=\sqrt{E_{S T}} h_{r_{t}} s+n_{r_{t}}+y_{I_{r_{t}}}
$$

where $y_{I_{r_{t}}}$ is the interference signal from the primary user due to missed-detection. The signal received at the $i$ th relay now becomes

$$
y_{i_{t}}=\sqrt{E_{S T}} h_{i_{t}} s+n_{i_{t}}+y_{I_{i_{t}}} .
$$

Following the same procedure as before, the received signal from the $i$ th relay can be written as

$$
y_{r_{i}}=\sqrt{\frac{E_{S T} E_{i}}{E_{S T}\left|h_{i_{t}}\right|^{2}+\sigma_{n_{i_{t}}}^{2}}} h_{r_{i}} h_{i_{t}} s+\hat{n_{r_{i}}}+y_{I_{r_{i}}}
$$

where $\hat{n_{r_{i}}} \sim C N\left(0, \sigma_{n_{r_{i}}}^{2}\right)$,

$$
\sigma_{n_{r_{i}}}^{\hat{2}}=\frac{E_{i}\left|h_{r_{i}}\right|^{2} \sigma_{n_{i_{t}}}^{2}}{E_{S T}\left|h_{i_{t}}\right|^{2}+\sigma_{n_{i_{t}}}^{2}}+\sigma_{n_{r_{i}}}^{2}
$$

and

$$
\hat{y}_{I_{i}}=y_{I_{r_{i}}}+\sqrt{\frac{E_{i}}{E_{S T\left|h_{i_{t}}\right|^{2}+\sigma_{n_{i_{t}}}^{2}}}} h_{r_{i}} y_{I_{i_{t}}} .
$$

Again, in matrix notation one has

$$
\mathbf{y}_{I}=\mathbf{h}_{I} s+\mathbf{n}_{I}+\mathbf{Y}_{I}
$$

where

$$
\begin{aligned}
& \mathbf{y}_{I}=\left[\frac{1}{\sigma_{n_{r_{t}}}} y_{r_{t}} \frac{1}{\sigma_{n_{r_{1}}}} y_{r_{1}} \ldots \ldots \ldots \frac{1}{\sigma_{n_{r_{m}}}} y_{r_{m}}\right]^{T} \\
& \mathbf{h}_{I}=\left[\sqrt{\frac{E_{S T}}{\sigma_{n_{r_{t}}}^{2}}} h_{r_{t}} \sqrt{\frac{E_{S T} E_{1}}{\sigma_{n_{r_{1}}}^{\hat{2}}\left(E_{S T}\left|h_{1_{t}}\right|^{2}+\sigma_{n_{1_{t}}}^{2}\right)}} h_{r_{1}} h_{1_{t}} \ldots\right. \\
& \left.\sqrt{\frac{E_{S T} E_{m}}{\sigma_{n_{r_{m}}}^{\hat{2}}\left(E_{S T}\left|h_{m_{t}}\right|^{2}+\sigma_{n_{m_{t}}}^{2}\right)}} h_{r_{m}} h_{m_{t}}\right]^{T} \\
& \mathbf{Y}_{I}=\left[\frac{1}{\sigma_{n_{r_{t}}}} y_{I_{r_{t}}} \frac{1}{\sigma_{n_{r_{1}}}} y_{I_{1}} \ldots \ldots \ldots \frac{1}{\sigma_{n_{r_{m}}}} y \hat{I}_{m}\right]^{T}
\end{aligned}
$$

\section{Spectrum Sensing}

Let $T$ denote the duration of one time slot. The source will employ spectrum sensing for duration of $t_{s}<T$ seconds. Transmission will take place, in the presence of a spectral hole, in the remaining time. An energy detector can be used for spectrum sensing. According to [7], the probabilities of detection and false alarm are given by

$$
\begin{gathered}
P_{d}=Q\left(\frac{\lambda-N-\gamma}{\sqrt{2(N+2 \gamma)}}\right) \\
P_{f}=Q\left(\frac{\lambda-N}{\sqrt{2 N}}\right)
\end{gathered}
$$

respectively, where $\lambda$ is the threshold of the energy detector, $N=t_{s} f_{s}$ is the number of samples, $f_{s}$ is the sampling frequency, $\gamma$ equals $N$ times the $S N R$ at the output of the detector and $Q($.$) is the Gaussian Q$-function. 


\section{OPTIMIZATION}

In this section, we present two optimal resource allocation algorithms to maximize throughput and SCR. We derive the throughput and SCR and quantify the constraints on the two optimization problems.

\section{A. Throughput}

Using our assumptions, we can derive the throughput as

$$
\begin{aligned}
C= & P\left(H_{0}\right) \log _{2}(1+S N R)\left(\frac{T-t_{s}}{T+m\left(T-t_{s}\right)}\right)\left(1-P_{f}\right)+ \\
& P\left(H_{1}\right) \log _{2}(1+S I N R)\left(\frac{T-t_{s}}{T+m\left(T-t_{s}\right)}\right)\left(1-P_{d}\right)
\end{aligned}
$$

where $S N R$ is as defined previously, $S I N R$ is the signalto-interference-noise-ratio, $P\left(H_{0}\right)$ is the probability that the primary user is absent, $P\left(H_{1}\right)=1-P\left(H_{0}\right)$ is the probability that the primary user is present and $\frac{T-t_{s}}{T+m\left(T-t_{s}\right)}$ is introduced as a penalty in throughput because out of the total time of $T+m\left(T-t_{s}\right)$, only $T-t_{s}$ data is transmitted. It can be shown that the $S N R$ can be written as

$$
S N R=a+\sum_{i=1}^{m} \frac{b_{i}}{\sigma_{n_{r_{i}}}^{\tilde{2}}}
$$

where

$$
a=\frac{E_{S T}\left|h_{r_{t}}\right|^{2}}{\sigma_{n_{r_{t}}}^{2}} \quad b_{i}=\frac{E_{S T} E_{i}\left|h_{r_{i}}\right|^{2}\left|h_{i_{t}}\right|^{2}}{E_{S T}\left|h_{i_{t}}\right|^{2}+\sigma_{n_{i_{t}}}^{2}} .
$$

The $S I N R$ can be expressed as

$$
S I N R=\frac{\left(a+\sum_{i=1}^{m} \frac{b_{i}}{\sigma_{n_{r_{i}}}^{2}}\right)^{2}}{a+\sum_{i=1}^{m} \frac{b_{i}}{\sigma_{n_{r_{i}}}^{2}}+\frac{a c}{\sigma_{n_{r_{t}}}^{2}}+\sum_{i=1}^{m} \frac{d_{i}}{\sigma_{n_{r_{i}}}^{2}} \frac{b_{i}}{\sigma_{n_{r_{i}}}^{\hat{2}}}}
$$

where

$$
\begin{aligned}
& c=E\left[\left|y_{I_{r_{t}}}\right|^{2}\right] \\
& d_{i}=E\left[\left|\hat{y}_{i}\right|^{2}\right] \\
& E\left[\left|\hat{y}_{I_{i}}\right|^{2}\right]=E\left[\left|y_{I_{r_{i}}}\right|^{2}\right]+E\left[\left|y_{I_{i_{t}}}\right|^{2}\right]\left(\frac{E_{i}}{\left.E_{S T \mid h_{i_{t}}}\right|^{2}+\sigma_{n_{i_{t}}}^{2}}\left|h_{r_{i}}\right|^{2}\right) .
\end{aligned}
$$

Substituting (11) and (12) in (10), gives the throughput as

$$
\begin{aligned}
& C=P\left(H_{0}\right) \log _{2}\left(1+a+\sum_{i=1}^{m} \frac{b_{i}}{\sigma_{n_{r_{i}}}^{2}}\right)\left(\frac{T-t_{s}}{T+m\left(T-t_{s}\right)}\right) \\
& \times\left(1-Q\left(\frac{\lambda-N}{\sqrt{2 N}}\right)\right)+P\left(H_{1}\right)\left(\frac{T-t_{s}}{T+m\left(T-t_{s}\right)}\right) \times \\
& \log _{2}\left(1+\frac{\left(a+\sum_{i=1}^{m} \frac{b_{i}}{\sigma_{n_{r_{i}}}^{2}}\right)^{2}}{a+\sum_{i=1}^{m} \frac{b_{i}}{\sigma_{n_{r_{i}}}^{2}}+\frac{a c}{\sigma_{n_{r_{t}}}^{2}}+\sum_{i=1}^{m} \frac{d_{i}}{\sigma_{n_{r_{i}}}^{2}} \frac{b_{i}}{\sigma_{n_{r_{i}}}^{2}}}\right) \\
& \times\left(1-Q\left(\frac{\lambda-N-\gamma}{\sqrt{2(N+2 \gamma)}}\right)\right) .
\end{aligned}
$$

Assuming unit symbol time, $E_{S T}$ and $E_{i}$ can be thought of as source and relay power, respectively. The constraints on the problem are therefore given by

$$
\begin{aligned}
& 0 \leq E_{S T} \leq E_{T}, \quad 0 \leq E_{i} \leq E_{i}^{\max }, \\
& E_{S T}+\sum_{i=1}^{m} E_{i} \leq E_{\text {total }} \text { and } P_{d} \geq P_{d}^{t h}
\end{aligned}
$$

where $P_{d}^{t h}$ specifies the constraint on $P_{d}, E_{T}$ is the power available at the source, $E_{i}^{\max }$ is the power available at the relays and $E_{\text {total }}$ is the total power available to the whole system. We have put a constraint on $P_{d}$ to restrict interference to the primary user.

\section{B. SCR}

To optimize the SCR, the value of symbol time, $T_{s}$, is also considered as a variable of optimization since the $S N R$ and $S I N R$ depend on the symbol time. As such, in order to have a trade off between sensing time and SCR, $T_{s}$ must be varied. The signal energies can now be written as

$$
E_{S T}=p_{S T} T_{s} \text { and } E_{i}=p_{i} T_{S}
$$

where $p_{S T}$ and $p_{i} \mathrm{~s}$ are the source and relay powers, respectively. The SCR is given by

$$
\begin{gathered}
S C R=P\left(H_{0}\right)(1-Q(\sqrt{k S N R}))\left(1-P_{f}\right)+ \\
P\left(H_{1}\right)(1-Q(\sqrt{k S I N R}))\left(1-P_{d}\right)
\end{gathered}
$$

where the constant $k$ depends on the constellation used. Putting (11), (12) and (15) into (16) gives

$$
\begin{array}{r}
S C R=P\left(H_{0}\right) \quad\left(1-Q\left(\sqrt{k\left(\alpha+\sum_{i=1}^{m} \frac{\beta_{i}}{\sigma_{n_{r_{i}}}^{\tilde{2}}}\right)}\right) \times\right. \\
\left(1-Q\left(\frac{\lambda-N}{\sqrt{2 N}}\right)\right)+P\left(H_{1}\right)\left(1-Q\left(\frac{\lambda-N-\gamma}{\sqrt{2(N+2 \gamma)}}\right)\right) \\
\left(\sqrt{\left.\frac{k\left(\alpha+\sum_{i=1}^{m} \frac{\beta_{i}}{\sigma_{n_{r_{i}}}^{2}}\right)^{2}}{\alpha+\sum_{i=1}^{m} \frac{\beta_{i}}{\sigma_{n_{n_{i}}}^{2}}+\frac{\alpha c}{\sigma_{n_{r_{t}}}^{2}}+\sum_{i=1}^{m} \frac{d_{i}}{\sigma_{n_{r_{i}}}^{2}} \frac{\beta_{i}}{\sigma_{n_{r_{i}}}^{2}}}\right)}\right)
\end{array}
$$

where

$$
\alpha=\frac{p_{S T} T_{s}\left|h_{r_{t}}\right|^{2}}{\sigma_{n_{r_{t}}}^{2}} \quad \beta_{i}=\frac{p_{S T} p_{i} T_{s}^{2}\left|h_{r_{i}}\right|^{2}\left|h_{i_{t}}\right|^{2}}{p_{S T} T_{s}\left|h_{i_{t}}\right|^{2}+\sigma_{n_{i_{t}}}^{2}} .
$$

We have the same power constraints as we had in the the throughput case discussed in the (14). In addition, we put a constraint on the bit rate, i.e. that at least $R$ symbols should be sent in one $T \mathrm{~ms}$ time slot. Now the constraints on the problem are given by

$$
\begin{aligned}
& 0 \leq p_{S T} \leq E_{T}, 0 \leq p_{i} \leq E_{i}^{\max }, P_{d} \geq P_{d}^{t h} \\
& p_{S T}+\sum_{i=1}^{m} p_{i} \leq E_{\text {total }}, \quad T_{s} \leq \frac{T}{R} \text { and } R T_{s}+t_{s} \leq T .
\end{aligned}
$$




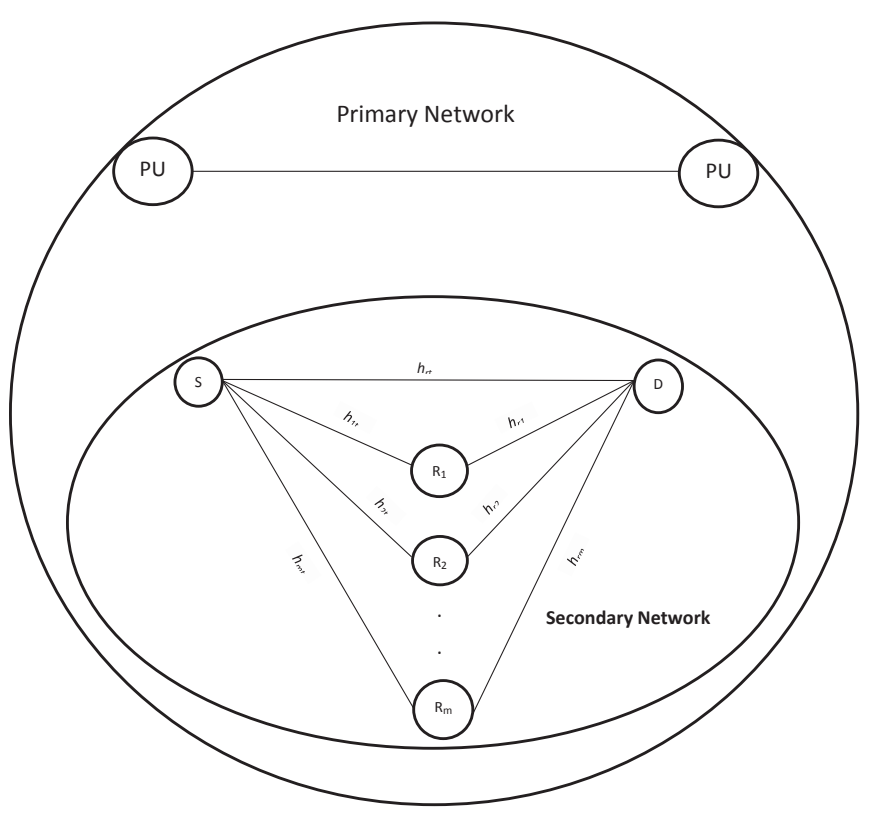

Fig. 1: Primary and secondary networks.

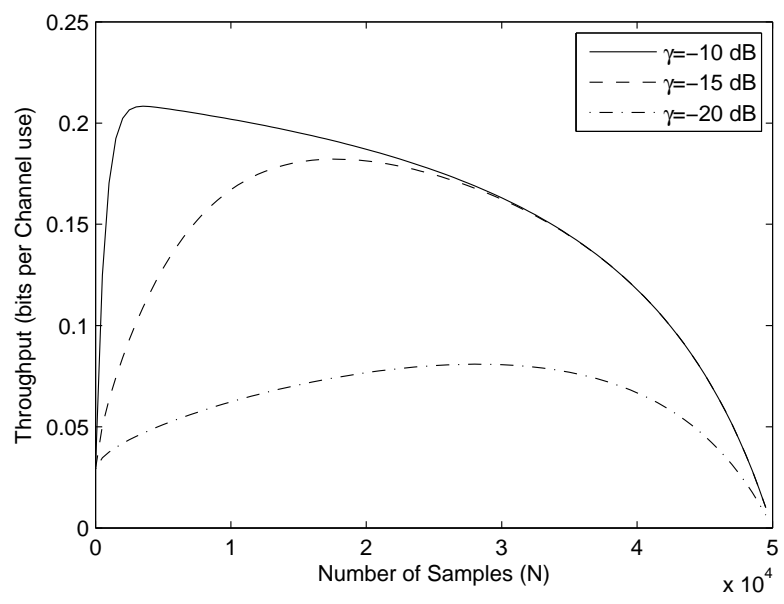

Fig. 2: Sensing-throughput trade off for different values of $\gamma$ at $m=4$.

\section{Numerical Results ANd Discussion}

In this section, we provide some selected numerical results for the optimization problems. We consider different system parameters and show that there exist optimal values for the sensing time and powers for maximizing throughput and SCR. All noise variances are set at $1 . E_{T}$ and $E_{i}^{\max }$ are 2, while $E_{\text {total }}$ is set at 4 except when results are shown against $m$, where it is set at $2+1.5 \mathrm{~m}$. All the channel coefficients were also taken to be one unless stated otherwise. The total frame duration $T$ is set as $100 \mathrm{~ms}$. We set $P_{d}^{t h}=0.9$, and $R=100$. For SCR, we assume binary phase shift keying (BPSK) signal. As there is a linear relationship between the number of samples and the sensing time, we here plot the number of samples

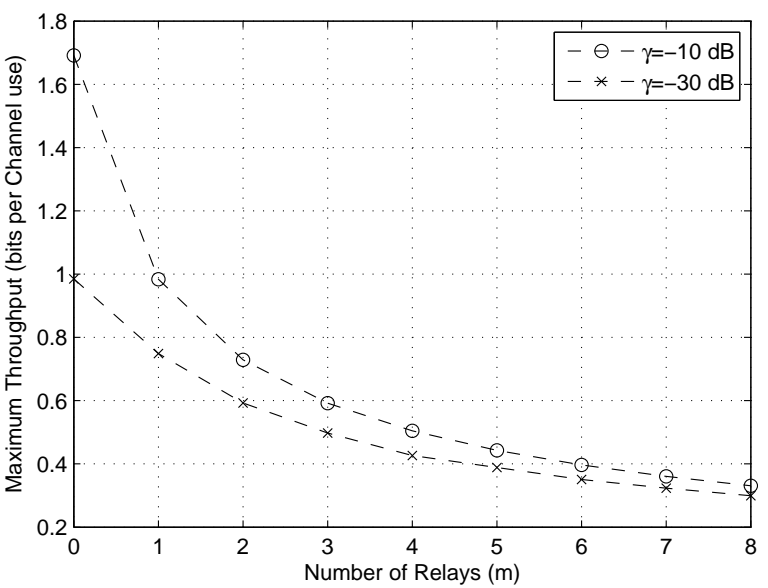

Fig. 3: Maximum achievable throughput as a function of the number of relays for different values of $\gamma$.

instead of the sensing time.

Figure 2 shows the achievable throughput as a function of the number of samples $N$ for different values of the primary SNR, $\gamma$. As one sees, there is an optimal value of $N$ where the achievable throughput is maximized. If $N$ is too low $P_{f}$ will increase, hence reduced throughput. If it is too high, the fraction of time for transmission will decrease, thus reducing throughput. The optimal value of the $N$ increases with decreasing $\gamma$. This is due to the fact that for a smaller $\gamma$, to achieve the same probability of detection larger number of samples is required. The maximum value of throughput also decreases as more time is spent on sensing, leaving less time for transmission.

The effect of the number of relays, $m$, on the maximum achievable throughput and optimal value of $N$ and the optimal source power is shown in Figures 3 and 4respectively. As expected, the throughput decreases with an increase in the number of relays as more time is required in sending one frame. The throughput also decreases with decreasing $\gamma$ as lower value of $\gamma$ implies greater sensing time and less transmission time. The optimal number of samples increases as the number of relays is increased as can be seen from Figure 4 . This is due to the $\frac{T-t_{s}}{T+m\left(T-t_{s}\right)}$ term. As $m$ is increased, the denominator increases. Hence, to compensate for this increase, the sensing time increases, i.e. $N$ increases, to reduce the denominator.

Figure 5 shows the optimal power allocation as a function of the channel response. The better the channel response, more power is allocated to it. The source power is the greatest as noted. The source power reaches its peak above a certain value of the channel coefficient. Figure 5 also shows that relay power allocation is more dependent on the channel between the relay and the source than the channel between the relay and the destination.

Figure 6 shows the trade-off in SER as a function of the symbol time $T_{s}$. This trade-off exists due to the constraint 


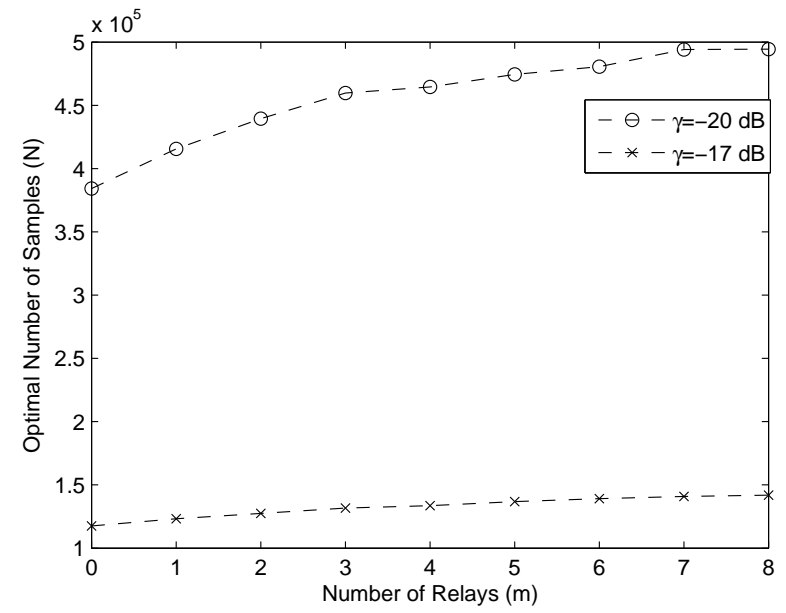

Fig. 4: Optimal number of samples as a function number of relays.

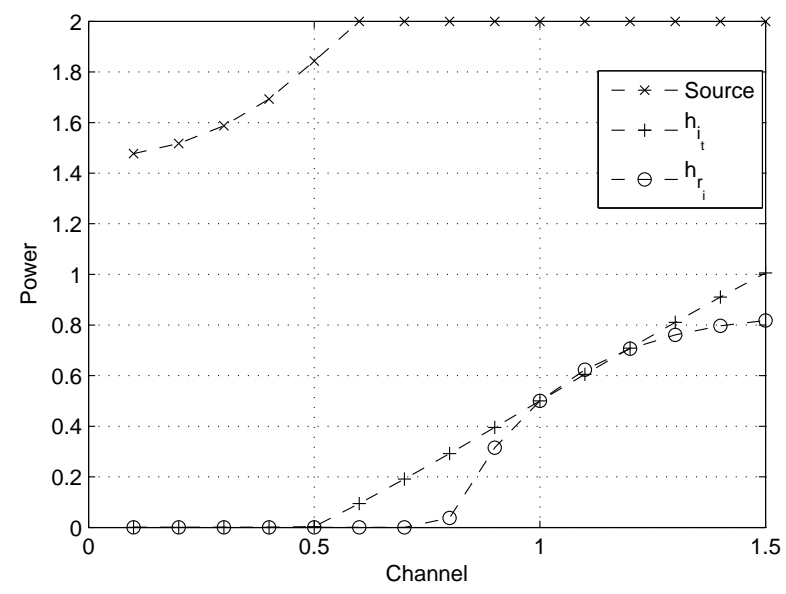

Fig. 5: Optimal power allocation versus Channel.

relating $t_{s}$ and $T_{s}$.

In Figure 7, we show the variation of the SCR with $m$. The figure shows a small increase in SCR with larger $m$. This is due to increase in $S N R$ and $S I N R$ with increasing $m$. An important point to note here is that the SCR and symbol error rate (SER) are not linearly related. Thus increase in SCR does not imply an equivalent decrease in SER. The SCR also increases with increasing $\gamma$, as higher $\gamma$ implies greater $T_{s}$.

\section{Conclusions}

Two optimal resource allocation schemes that maximize throughput and SCR have been proposed. Numerical Results show that optimal values of the sensing time, source power, and relay powers exist that maximize the targeted parameters. It has also been shown that the optimal power allocation is independent of the primary $S N R, \gamma$ and the number of relays, $m$ but is dependent on the channel between source and destination, source and relays, relays and destination. In addition, in power allocation for relays, the channel between the relay and source is of greater significance than the channel between relay and destination.

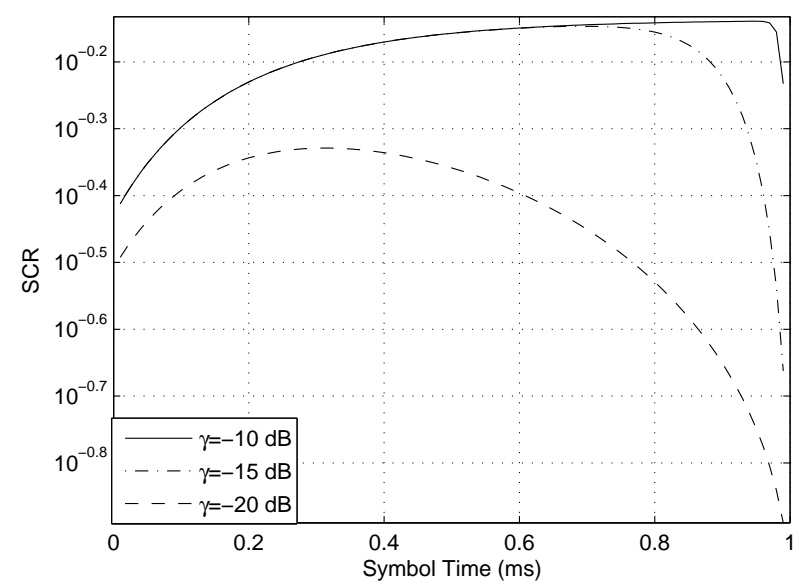

Fig. 6: Variation in SCR with respect to the symbol time with $m=4$.

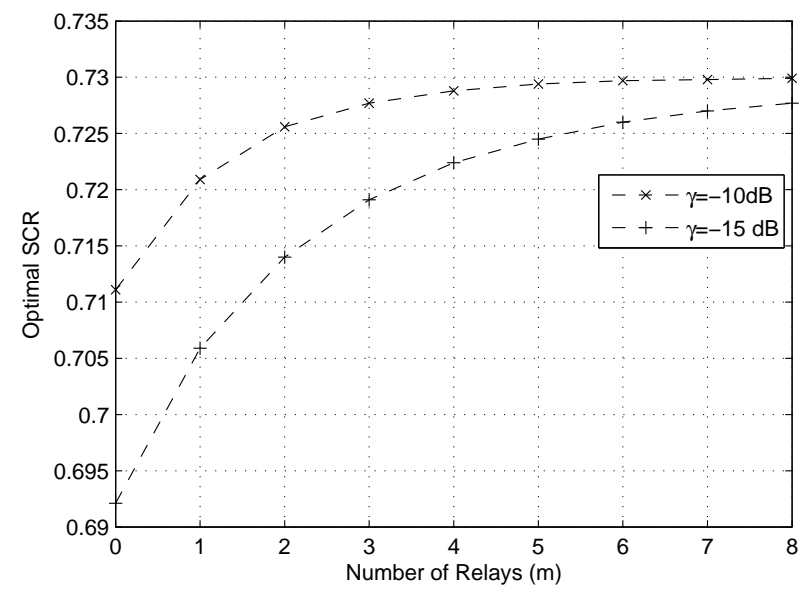

Fig. 7: Optimal SCR as a function of number of relays.

\section{REFERENCES}

[1] O. Simeone, J. Gambini, Y. Bar-Ness, and U. Spagnolini, "Cooperation and cognitive radio," in Proc. IEEE International Conference on Communications 2007 (ICC 2007), Glasgow, Scotland, UK, Jun. 2007, pp. $6511-6515$.

[2] Y. Zou, Y. Yao, and B. Zheng, "Cognitive transmissions with multiple relays in cognitive radio networks," IEEE Transactions on Wireless Communications, vol. 10, no. 2, pp. 648-659, Feb. 2011.

[3] Y. Liang, Y. Zeng, E. C. Y. Peh, and A. T. Hoang, "Sensing-throughput tradeoff for cognitive radio networks," IEEE Transactions on Wireless Communications, vol. 7, no. 4, pp. 1326-1337, Apr. 2008.

[4] L. Li, X. Zhou, H. Xu, G. Y. Li, D. Wang, and A. Soong, "Simplified relay selection and power allocation in cooperative cognitive radio systems," IEEE Transactions on Wireless Communications, vol. 10, no. 1, pp. 3336, Jan. 2011.

[5] L. Jayasinghe and N. Rajatheva, "Optimal power allocation for relay assisted cognitive radio networks," in IEEE 72nd Vehicular Technology Conference Fall 2010 (VTC 2010-Fall), Ottawa, Canada, Sep. 2010, pp. $1-5$.

[6] Z. Shu and W. Chen, "Optimal power allocation in cognitive relay networks under different power constraints," in IEEE International Conference on Wireless Communications, Networking and Information Security 2010 (WCNIS 2010), Beijing, China, Jun. 2010, pp. 647-652.

[7] H. Urkowitz, "Energy detection of unknown deterministic signals," Proc. IEEE, vol. 55, no. 4, pp. 523-531, Apr. 1967. 